\title{
Preoperative evaluation of micro-organisms in non-operated cleft in soft palate: impact on use of antibiotics
}

\author{
G.J. Roode ${ }^{a, *}$, K.-W. Bütow ${ }^{b, c, d}$, S. Naidoo ${ }^{b}$ \\ ${ }^{a}$ Department of Anatomy and Department of Maxillo-Facial and Oral Surgery, University of Pretoria, \\ P/Bag x 323, Arcadia 0007, South Africa \\ ${ }^{b}$ Department of Maxillo-Facial and Oral Surgery, University of Pretoria, PO Box 1266, Pretoria 0001, \\ South Africa \\ c University of KwaZulu-Natal, Durban, South Africa \\ ${ }^{d}$ The Wilgers Hospital, Pretoria, South Africa \\ * Corresponding author. Tel.: +27 12319 2234; Fax: +27 866938067. \\ E-mail addresses: giel.roode@up.ac.za, giel.roode@gmail.com (G.J. Roode), kurt@butow.co.za (K.- \\ W. Bütow), shan.naidoo@up.ac.za (S. Naidoo).
}

\begin{abstract}
To identify the pathogenic micro-organisms that had colonised preoperatively in clefts in the soft palate and oro-nasopharynx, we retrospectively studied the preoperative microbiological profiles of 200 infants who had had primary repair of all types of cleft in the soft palate. Data from a private practice that specialises in the repair of facial clefts were extracted randomly from patients' files. We analysed the results of the culture of preoperative swabs taken from clefts in the soft palate and oro-nasopharynx, and the resistance profile of organisms towards various antibiotics. A total of 23 different pathogenic micro-organisms were isolated from 115 (57\%) of the sample. Klebsiella pneumoniae most commonly colonised clefts in the lip, alveolus, and palate. This was considerably higher than in other groups. The second most common micro-organism was Staphylococcus aureus, which was found most often in patients with isolated clefts in the hard palate. Those with complete cleft lip and palate presented with more pathogenic micro-organisms in preoperative cultures than those with other types of cleft. We need to find a way to control pathogenic micro-organisms in the oral and oro-nasopharyngeal region preoperatively to limit postoperative complications.
\end{abstract}

\section{Keywords}

Cleft palate; Micro-organisms; Antibiotics; Resistance; Oro-nasal fistula 


\author{
Abbreviations \\ $\mathrm{CP}$ - cleft palate \\ CLP - cleft lip and palate \\ CLAP - cleft lip, alveolus and palate \\ bCLAP - bi-lateral cleft lip, alveolus and palate \\ UCLAP - uni-lateral cleft lip, alveolus and palate \\ $\mathrm{COMBI}$ - cleft lip + cleft hard palate or soft palate \\ hPsP - cleft hard palate and soft palate \\ sP - cleft soft palate
}

\title{
Introduction
}

A report from 1937 states that infection is the most common reason why operations fail. ${ }^{1}$ Clinicians constantly evaluate the role of microbial infections related to complications after the repair of clefts in the soft palate, and the authors of two studies, which reported the presence of Staphylococcus aureus in unrepaired clefts in the lip and palate,,$\frac{2,3}{3}$ concluded that it inhabits the nasopharyngeal and nasal cavity and colonises the oral cavity through the cleft. In a study of 100 infants, 15 different pathogenic organisms were identified in the oro-nasopharyngeal cavity of patients with a cleft lip and palate and those with a cleft palate $(C P)$ during the perioperative stage of primary palatal repair. ${ }^{4}$

In our unit between 1984 and March 2015, 4183 patients were treated for facial clefts. Of them, 3261 (78\%) had a cleft in the soft palate, 1597 (38\%) a cleft lip, alveolus and palate (CLAP), 715 (17\%) a cleft in the hard and soft palate (hPsP), 773 (19\%) an isolated cleft in the soft palate (sP), and $171(4 \%)$ a COMBI cleft (CL + hPsP or sP). The protocol for repair starts with primary reconstruction of the cleft in the soft palate.

Pathogenic micro-organisms that are found preoperatively might influence outcome. After a search of PubMed, Ovid, and EBSCOhost, we found 12 publications on the oral flora in infants and toddlers with clefts, seven of which identified micro-organisms found preoperatively. $\frac{2-8}{-}$ One compared the oral types of micro-organisms found in relation to the type of cleft,, four referred to Streptococcus mutans and lactobacilli, which are involved in dental caries, but do not apply in infants,,$\frac{6,9-11}{2}$ and one analysed the prevalence and composition of bacteraemia associated with operations to repair a cleft lip and palate. ${ }^{12}$ Others compared the association of postoperative complications with pathogenic organisms found perioperatively. $\frac{3,4,7,8}{}$

With the misuse of antimicrobials in mind, we have therefore reported the variety of species of micro-organisms found in different types of cleft in the posterior soft palate and oronasopharyngeal region before primary repair.

\section{Patients and methods}

This retrospective study was designed and approved by the Faculty of Health Sciences Research Ethics Committee (297/2014) of the University of Pretoria. Data were randomly 
collected from the files of 3261 infants (aged between 5 and 7 months) who were treated between January 1992 and March 2015. A total of 200 patients who had had primary repair of clefts in the soft palate were included. They had been admitted to the hospital from all over the country, and a paediatrician had deemed them healthy before the operation. To ensure reliability, one researcher selected every sixteenth file that contained all the inclusion criteria, and recorded all the data. All patients had had microbial swabs taken preoperatively from clefts in the soft palate and oro-nasopharyngeal region after induction of anaesthesia and orotracheal intubation, but before they had been surgically prepared. During the operation, a nasogastric tube was inserted for postoperative feeding for six days to prevent contamination of the wound. Antimicrobial drugs given postoperatively were adjusted as soon as the sensitivity results were available. Data on the type of cleft, type of micro-organisms cultured and identified from preoperative swabs, and the report of the sensitivity of micro-organisms to specific antimicrobials, were recorded on a Microsoft Access Database and Excel spread sheet. Statistical tests used was ANOVA (Analyses of variance) between the different groups.

\section{Results}

We matched the microbiological reports with the type of cleft, and divided the patients into four groups according to the type: patients with an isolated cleft in the soft palate (sP group, $n=52$ ), those with clefts in the hard and soft palate (hPsP group, $n=63$ ); those with clefts in the lip, alveolus, and hard and soft palate (CLAP group), who were subdivided into unilateral $(n=54)$, and bilateral $(n=22)$ groups; and those with a CL and hPsP or SP (COMBI group, $n=9)$.

A total of 23 different pathogenic micro-organisms were isolated from 115 patients (58\%) $(p=0.003)$. In the remaining $85(43 \%)$, oral flora was normal. Table 1 shows the pathogens identified, and Table 2 the pathogens in the different groups. Fig. 1 shows the pathogens found in the SP and hPsP groups, and Fig. 2 those found in the unilateral and bilateral CLAP groups and the COMBI group. Fig. 3 shows the anti-microbial resistant profile of the seven most common pathogens. We compared the largest number cultured in 10 or more patients using the layout of previously published resistance profiles of the perioperative organisms. ${ }^{4}$

Table 1. Pathogens identified in 115 patients.

\section{Pathogen No. of patients}

$\begin{array}{ll}\text { Klebsiella pneumoniae } & 35 \\ \text { Staphylococcus aureus } & 25 \\ \text { Escherichia coli } & 20 \\ \text { Streptococcus pneumoniae } & 14 \\ \text { Haemophilus influenzae } & 13 \\ \text { Moraxella catarrhalis } & 12 \\ \text { Enterobacter cloacae } & 10 \\ \text { Serratia marcescens } & 8 \\ \text { Acinetobacter baumannii } & 4 \\ \text { Enterobacter agglomerans } & 4 \\ \text { Pseudomonas aeruginosa } & 4\end{array}$




\section{Pathogen No. of patients}

Alpha-haemolytic streptococcus 3

Klebsiella oxytoca 3

Aeromonas hydrophila 2

Enterobacter aerogenes $\quad 2$

Aeromonas sobria 1

Enterobacter gergoviae $\quad 1$

Enterococcus faecalis 1

Kluyvera cryocrescens 1

Staphylococcus epidermidis 1

Streptococcus anginosus $\quad 1$

Streptococcus pyogenes 1

Streptococcus viridans $\quad 1$

Table 2. Cleft groups: normal compared with pathogenic micro-organisms.

Group Total $p$ value

$s P p$ value $h P s P p$ value uCLAP $p$ value bCLAP $p$ value COMBI $p$ value

\begin{tabular}{|c|c|c|c|c|c|c|c|c|c|c|}
\hline Normal & 26 & 31 & & 19 & & 6 & & 3 & & 85 \\
\hline Pathogeni & c 261.00 & 32 & 0.87 & 35 & 0.002 & 16 & 0.003 & 6 & 0.16 & 115 \\
\hline Total & 52 & 63 & & 54 & & 22 & & 9 & & 200 \\
\hline
\end{tabular}

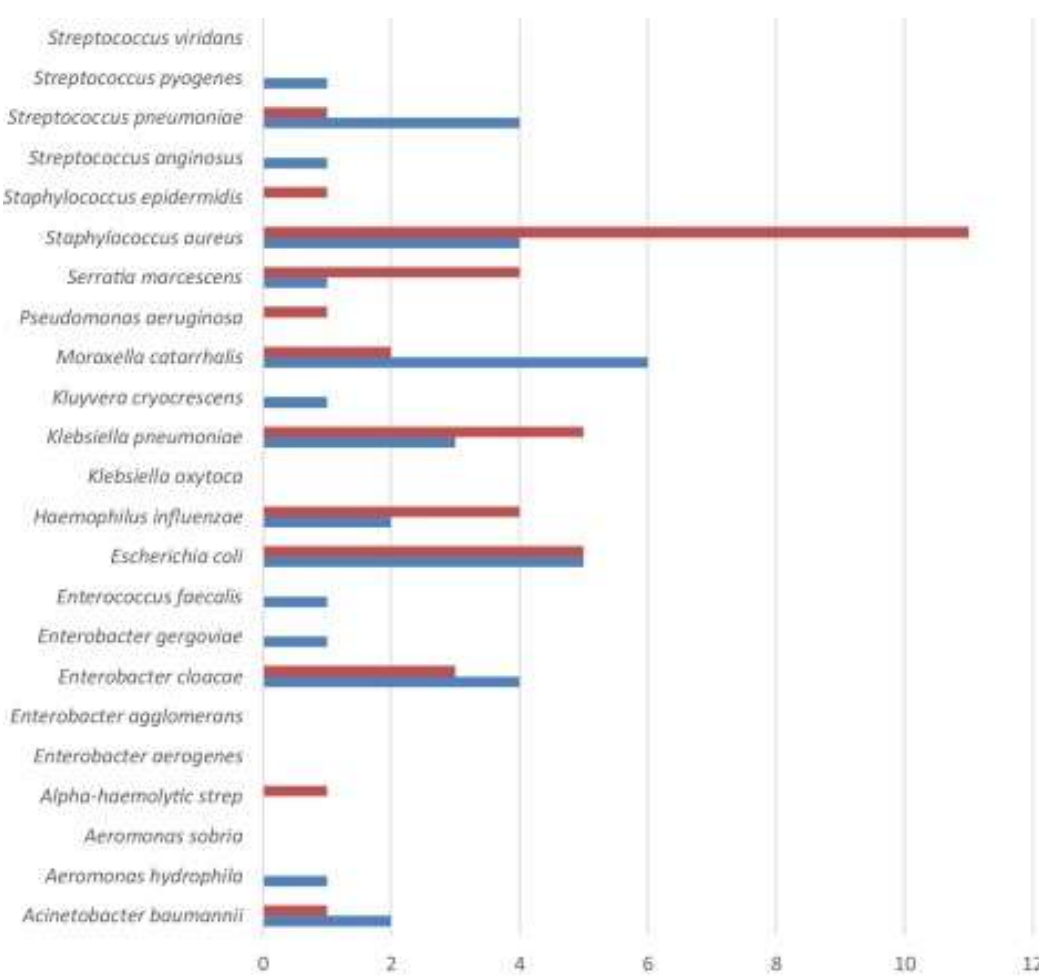

Fig. 1. Pathogenic micro-organisms found in clefts in the soft (blue) and hard (red) palate. 


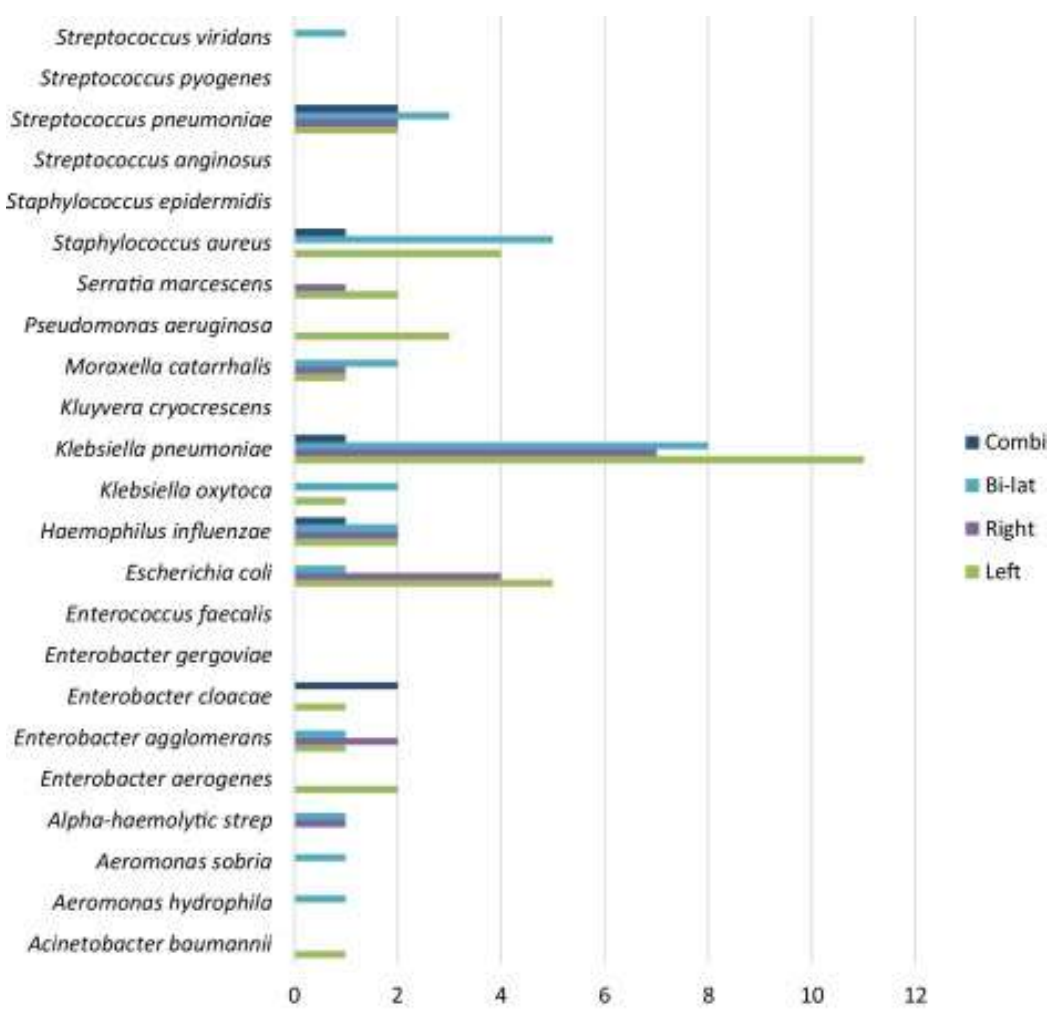

Fig. 2. Pathogenic micro-organisms found in unilateral and bilateral clefts in the lip, alveolus, and palate, and in clefts in the lip and hard and soft palate, or soft palate (COMBI).

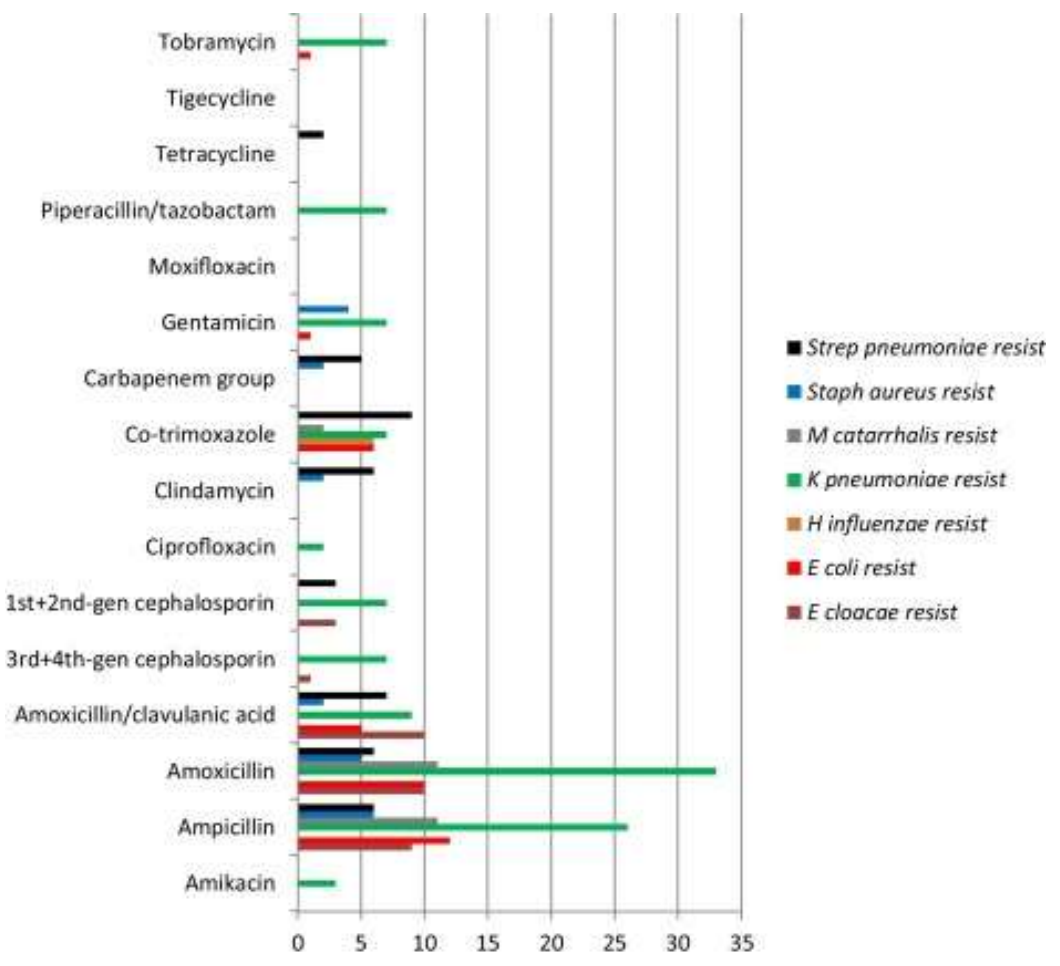

Fig. 3. Anti-microbial resistance profile. 


\section{Discussion}

We found only one publication that identified preoperative pathogenic micro-organisms in different types of cleft. $\stackrel{8}{-}$

In our group, pathogenic micro-organisms were found in 115 patients (58\%). Our finding that Klebsiella pneumoniae was the most common of the 23 different species reported ( $n=35 / 115,30 \%$ ), is similar to that of Cocco et al who compared two different types of

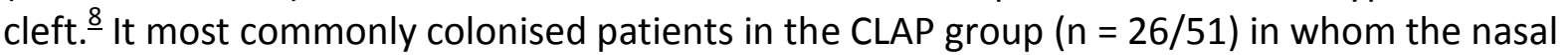
cavity was unilaterally or bilaterally exposed to the oral cavity. The second most common pathogen, Staphylococcus aureus, colonised 25 patients (22\%), of whom most were in the hPsP group ( $n=11 / 32$ ). In these infants, the cleft in the hard palate was partially exposed to the nasal cavity. The third most common, Escherichia coli, was found in 20 patients (17\%) (CLAP group: $n=10 / 51$; hPsP group: $n=5 / 32$; sP group: $n=5 / 26$ ). Four other microorganisms were identified in 10 or more patients: Streptococcus pneumoniae $(n=14)$, Haemophilus influenzae $(n=13)$, Moraxella catarrhalis $(n=12)$ and Enterobacter cloacae $(n=10)$. Serratia marcescens was found in eight. Seven pathogens found in two to four patients, and eight others in one patient each may be ignored, as they presented too infrequently (Table 1).

Klebsiella pneumoniae and Enterobacter cloacae are ever-present in the human respiratory and intestinal tracts. It may be hypothesised that infants aged between 5 and 7 months are more prone to those in the upper respiratory tract because of bottle-feeding or faecal or urinary contamination of the oral and oro-nasopharyngeal cavities, which is particularly likely when infection control by parents or carers is poor and infants put their hands in their mouths. Klebsiella pneumoniae is reported to be the most common nosocomial isolate that is a growing threat, as it produces beta-lactamase, most importantly carbapenemase,,$\underline{13,14}$ and our study has confirmed that it had the highest resistance to antimicrobials. Clinicians should therefore be aware of possible microbial incubation in a seemingly healthy infant, as antimicrobial resistance can contribute to postoperative infection. We found no record of Klebsiella pneumoniae resistance to carbapenemase.

The second most common pathogen recorded in 1997 was Staphylococcus aureus. $\frac{15}{\text { Around }}$ $20 \%$ of people are persistent carriers of one strain, and about $60 \%$ harbour it intermittently. Our results are within the same ranges as those published, $\underline{15}$ and most affected $(n=11)$ were in the hPsP group. As an increasing number of methicillin-resistant strains of Staphylococcus aureus (MRSA) have been identified, $\frac{8,15}{2}$ more and more postoperative complications may not respond to standard antimicrobials.

Seven complications had been recorded (four oronasal fistulas, and three infected wounds with hyperaemia), which compares favourably with other studies. ${ }^{4,16-23}$ Two of the fistulas had developed in patients who had no pathogenic micro-organisms in their preoperative swabs. In one, Serratia marcescens was cultured on day two from the swab, so a hospitalacquired infection might have caused the complication. In the other, five pathogenic organisms were found on day two (Alpha-haemolytic streptococcus, Enterobacter agglomerans, Enterococcus faecalis, Escherichia coli, and Staphylococcus aureus). The 
parents stayed with the infants in hospital and cared for them postoperatively, so we question whether they had maintained the standards of hygiene.

Of the five patients (two with fistulas and three with wound infection and hyperaemia) whose swabs showed pathogens, three presented with Klebsiella pneumoniae, one with the closely related Klebsiella oxytoca, and one with Enterobacter cloacae. These microbes, which were also isolated from swabs taken on day two before the antimicrobials had been adjusted, showed the highest resistance and were therefore associated with the complications. We assumed that the surgical technique did not have a role in the formation of any of the fistulas, as they were all associated with postoperative pathogens.

Before the operation, it is important to anticipate possible causes of postoperative infections by identifying potential pathogenic micro-organisms, particularly those resistant to antimicrobials. This can prevent or minimise complications after the primary repair of clefts in the soft palate and will limit the breakdown of wounds that can permanently impair speech.

We did this study with the misuse of antimicrobials in mind, and were alarmed by the number of pathogens cultured from preoperative swabs from infants only 5 months old that showed resistance. The results are worrying and a solution needs to be found where alternative (natural) methods can be used to control the presence of pathogens in the oronasopharyngeal region perioperatively.

\section{Conflict of interest}

We have no conflicts of interest.

\section{Funding source}

No funding was secured for this study.

\section{Financial disclosure}

All the authors have no financial relationships relevant to this article to disclose.

\section{Ethics statement/confirmation of patients' permission}

Clinical trial registration: Faculty of Health Sciences Research Ethics Committee of the University. The patients' parents gave their consent for us to obtain the data.

\section{References}

1. Kilner TP. Cleft lip and palate repair technique. London: St Thomas's Hospital Report; 1937127-40.

2. Arief EM, Mohamed Z, Idris FM. Study of viridans streptococci and Staphylococcus species in cleft lip and palate patients before and after surgery. Cleft Palate Craniofac J 2005;42:277-9. 
3. Rennie A, Treharne LJ, Richard B. Throat swabs taken on the operating table prior to cleft palate repair and their relevance to outcome: a prospective study. Cleft Palate Craniofac J 2009;46:275-9.

4. M"yburgh HP, Bütow KW. Cleft soft palate reconstruction: prospective study on infection and antibiotics. Int J Oral Maxillofac Surg 2009;38:928-32.

5. Chuo CB, Timmons MJ. The bacteriology of children before primary cleft lip and palate surgery. Cleft Palate Craniofac J 2005;42:272-6.

6. Bokhout B, van Loveren C, Hofman FX, et al. Prevalence of Streptococcus mutans and lactobacilli in 18-month-old children with cleft lip and/or palate. Cleft Palate Craniofac J 1996;33:424-8.

7. Thomas GP, Sibley J, Goodacre TE, et al. The value of microbiological screening in cleft lip and palate surgery. Cleft Palate Craniofac J 2012;49:708-13.

8. Cocco JF, Antonetti JW, Burns JL, et al. Characterization of the nasal, sublingual, and oropharyngeal mucosa microbiota in cleft lip and palate individuals before and after surgical repair. Cleft Palate Craniofac J 2010;47:151-5.

9. Antoszewska J, Kawala B, Minch L. Selected aspects of the oral environment in cleft palate patients - a problem evidently beyond dentists' scope. Postepy Hig Med Dosw (Online) 2010;64:659-64.

10. de Soet JJ, Bokhout B, Buijs JF, et al. Transmission of mutans streptococci between mothers and children with cleft lip and/or palate. Cleft Palate Craniofac J 1998;35:460-4.

11. van Loveren C, Buijs JF, Bokhout B, et al. Incidence of mutans streptococci and lactobacilli in oral cleft children wearing acrylic plates from shortly after birth. Oral Microbiol Immunol 1998;13:28691.

12. Adeyemo WL, Adeyemi MO, Ogunsola FT, et al. Prevalence and bacteriology of bacteremia associated with cleft lip and palate surgery. J Craniofac Surg 2013;24:1126-31.

13. Sumer S, Turk Dagi H, Findik D, et al. Two outbreaks of ESBL-producing Klebsiella pneumoniae in a neonatal intensive care unit. Pediatr Int2014;56:222-6.

14. Won SY, Munoz-Price LS, Lolans K, et al. Emergence and rapid regional spread of Klebsiella pneumoniae carbapenemase-producing Enterobac-teriaceae. Clin Infect Dis 2011;53:532-40.

15. Kluytmans J, van Belkum A, Verbrugh H. Nasal carriage of Staphylococcus aureus: epidemiology, underlying mechanisms, and associated risks. Clin Microbiol Rev 1997;10:505-20.

16. Amaratunga NA. Occurrence of oronasal fistulas in operated cleft palate patients. J Oral Maxillofac Surg 1988;46:834-7.

17. Shimizu M, Shigetaka $Y$, Mizuki $H$, et al. Oronasal fistulae in repaired cleft palates. J Craniomaxillofac Surg 1989;17:37-8.

18. Lees VC, Pigott RW. Early postoperative complications in primary cleft lip and palate surgeryhow soon may we discharge patients from hospital? Br J Plast Surg 1992;45:232-4. 
19. Losken HW, van Aalst JA, Teotia SS, et al. Achieving low cleft palate fistula rates: surgical results and techniques. Cleft Palate Craniofac J 2011;48:312-20.

20. Dec W, Shetye PR, Grayson BH, et al. Incidence of oronasal fistula formation after nasoalveolar molding and primary cleft repair. J Craniofac Surg 2013;24:57-61.

21. Deshpande GS, Campbell A, Jagtap R, et al. Early complications after cleft palate repair: a multivariate statistical analysis of 709 patients. J Craniofac Surg 2014;25:1614-8.

22. Hardwicke JT, Landini G, Richard BM. Fistula incidence after primarycleft palate repair: a systematic review of the literature. Plast ReconstrSurg 2014;134:618e-27e.

23. Zhang Z, Fang $S$, Zhang $Q$, et al. Analysis of complications in primary cleft lips and palates surgery. J Craniofac Surg 2014;25:968-71. 